\title{
A Multi-ASIC Real-Time Implementation of the Two Dimensional Affine Transform with a Bilinear Interpolation Scheme
}

\author{
MARK J. BENTUM, MARTIN M. SAMSOM AND CORNELIS H. SLUMP \\ University of Twente, Department of Electrical Engineering, Laboratory for Network Theory and VLSI Design, \\ P.O. Box 217, 7500 AE Enschede, The Netherlands
}

Received May 25, 1993; Revised October 19, 1994

\begin{abstract}
Some image processing applications (e.g. computer graphics and robot vision) require the rotation, scaling and translation of digitized images in real-time (25-30 images per second). Today's standard image processors can not meet this timing constraint so other solutions have to be considered. This paper describes a multi-ASIC solution which is capable of doing the image processing tasks in real-time. The first ASIC is a so-called affine transformer which calculates a one-dimensional coordinate every $25 \mathrm{~ns}$. The second ASIC is a bilinear interpolator which calculates an interpolated value from four known surrounding values, again every $25 \mathrm{~ns}$. This ASIC is designed in a modular setup which results in a flexible accuracy of the interpolation. If more accurate interpolation is required, another ASIC (containing an interpolation stage) is used. In this way for each application a proper accuracy is implemented, reaching optimal silicon area utilization and desired accuracy of interpolation. Using two affine transformers (for obtaining a two dimensional coordinate pair) and an interpolator, one can build a system which can translate, rotate and scale an image of size $1024 * 1024$ in real-time (25-30 images per second). In this paper the system as well as the design of the ASICs are presented.
\end{abstract}

\section{Introduction}

Digital image processing has a fast growing flow of applications [1], [2], mainly due to the strongly increasing performance of computers. However, realtime applications are still difficult to implement, so we have to look at dedicated solutions. Here we address the problem of the real-time manipulation of images. This means we want to rotate, translate and scale an image of size $1024 \times 1024$ at a rate of 25-30 images per second. Figure 1 shows an example of image manipulation.

This technique of manipulating an image in real-time can be used in many applications, like medical applications (e.g. real-time $X$-ray), industrial applications (e.g. robot vision) and also in consumer electronics (e.g. digital video camera).

This paper consists of six sections. Section 2, which follows this introduction, presents the mathematical background of the system. The efficient implementation of the algorithm in VLSI is discussed in section 3. In section 4 we discuss our design methodology. We consider this section to be very important because it will present a methodology which we think will result in a first-time-correct design. In section 5 the results will be shown. We end this paper with a discussion.

\section{Mathematical Background}

In this section the mathematical background of the problem is addressed. The system to be designed must be capable of rotating, translating and scaling an image. This process can be described as a coordinate transformation. If $(i, j)$ are the coordinates of a pixel in the input image and $(x, y)$ the corresponding coordinates in the output image, we can write the following matrix-equation describing the desired operations:

$$
\left(\begin{array}{l}
x \\
y
\end{array}\right)=\left(\begin{array}{ll}
A & B \\
C & D
\end{array}\right)\left(\begin{array}{l}
i \\
j
\end{array}\right)+\left(\begin{array}{l}
E \\
F
\end{array}\right)
$$

$A$ through $F$ are constants containing the information about the amount of rotation, translation and scaling. If the origin of the image is in the upper left corner, we can write the constants $A$ through $F$ as follows:

$$
\begin{aligned}
& A=S_{x} \cos \alpha \\
& B=-S_{x} \sin \alpha \\
& C=S_{y} \sin \alpha \\
& D=S_{y} \cos \alpha \\
& E=O_{x}\left(1-S_{x} \cos \alpha-S_{x} \sin \alpha\right)+X \\
& F=O_{y}\left(1-S_{y} \cos \alpha+S_{y} \sin \alpha\right)+Y
\end{aligned}
$$



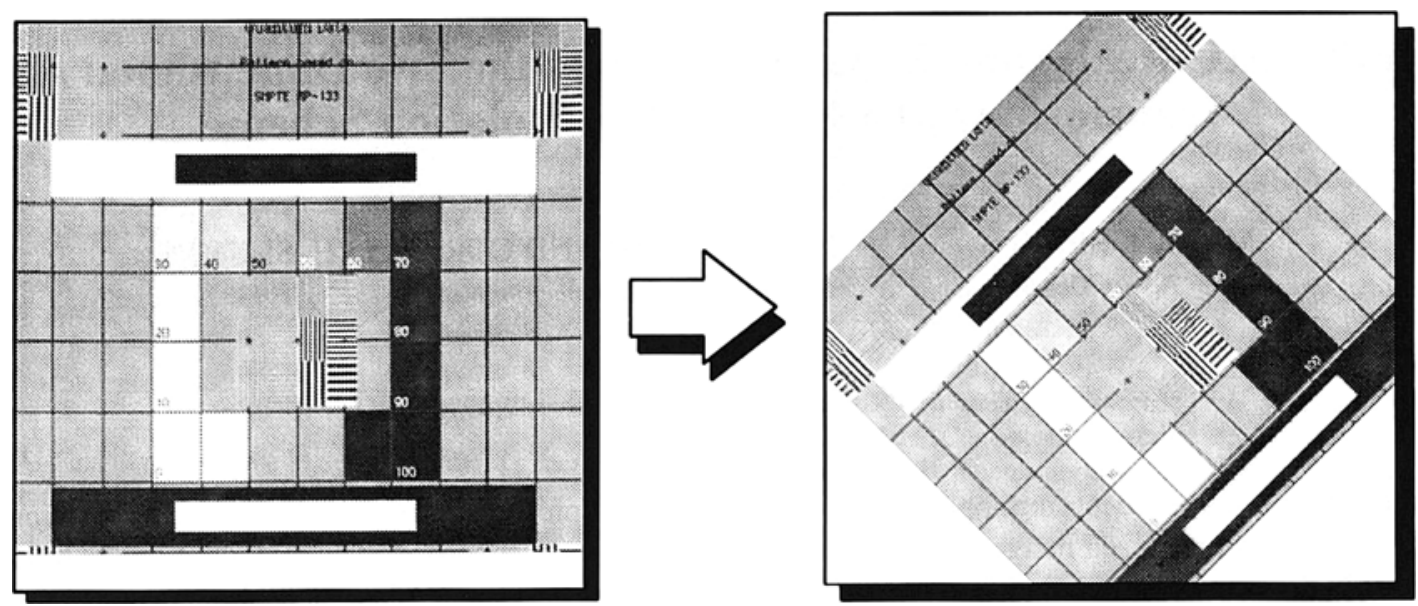

Fig. I. Example of the manipulation of an image.

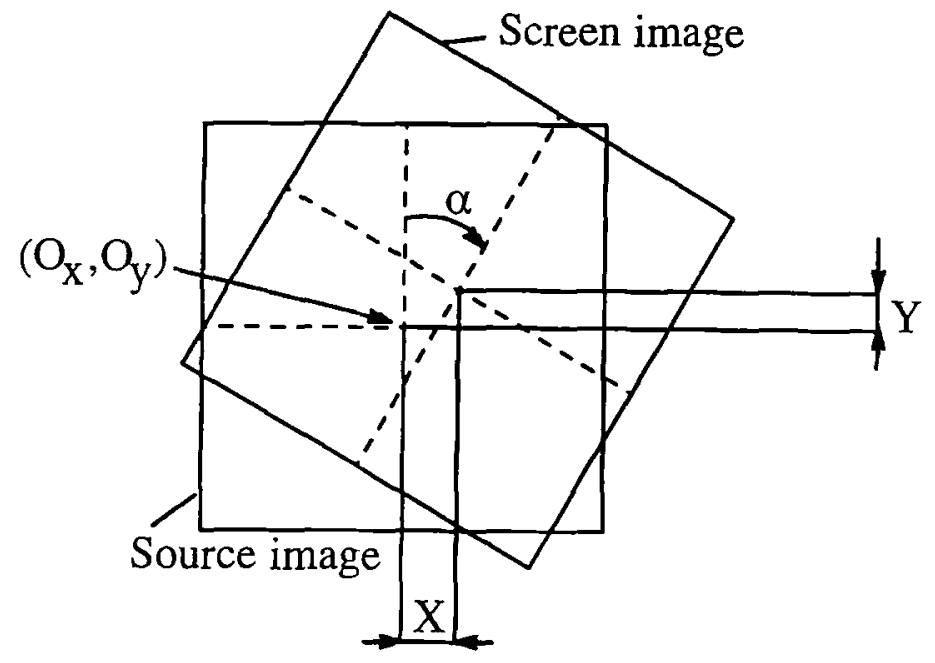

Fig. 2. An example of the parameters for image manipulation.

Here $\alpha$ is the counterclockwise rotation angle of the image (with the center of the image $\left(O_{x}, O_{y}\right)$ as the rotation-axis), $S_{x}, S_{y}$ are the scale factors and $X, Y$ are the pixel translation distances in $x$ and $y$ direction. In Fig. 2 an example for the parameters $\left(O_{x}, O_{y}\right), X$, $Y$, and $\alpha$ is given.

Some applications require the constants to be recalculated for every frame. Because the maximum frame rate is 30 frames per second, these 6 constants have to be calculated every $33 \mathrm{~ms}$. The calculation of these constants can easily be done by a general purpose processor (PC or low performance workstation). The actual image addresses however can not be computed in real-time with such a general purpose processor (see section 3).
In Eq. 1 the input image is scanned, yielding pixel information for the output image. This method is often referred to as source scanning. Source scanning is seldom used, since it results in holes in the output image because of overlapping pixels. Another disadvantage is the necessary implementation of four multiplications and two additions for every pixel in the input image, which would require a large VLSI area (when a VLSI implementation is used).

A more efficient method is screen scanning. In screen scanning the output image is scanned. In Eq. 3 the inverse transformation is given.

$$
\left(\begin{array}{l}
i \\
j
\end{array}\right)=\left(\begin{array}{ll}
A^{\prime} & B^{\prime} \\
C^{\prime} & D^{\prime}
\end{array}\right)\left(\begin{array}{l}
x \\
y
\end{array}\right)+\left(\begin{array}{l}
E^{\prime} \\
F^{\prime}
\end{array}\right)
$$


Again $(x, y)$ are the coordinates in the output image and $(i, j)$ are the coordinates in the input image, but now the input coordinates are calculated corresponding to a certain output coordinate pair. Of course the constants $A^{\prime}$ through $F^{\prime}$ are different constants than the constants in Eq. 1:

$$
\begin{aligned}
A^{\prime}= & \frac{\cos \alpha}{S_{x}} \\
B^{\prime}= & \frac{\sin \alpha}{S_{y}} \\
C^{\prime}= & \frac{-\sin \alpha}{S_{x}} \\
D^{\prime}= & \frac{\cos \alpha}{S_{y}} \\
E^{\prime}= & O_{x}\left(1-\frac{\cos \alpha}{S_{x}}\right)-X \frac{\cos \alpha}{S_{x}}-Y \frac{\sin \alpha}{S_{y}} \\
F^{\prime}= & O_{y}\left(1-\frac{\sin \alpha}{S_{y}}\right. \\
& \left.\quad+O_{x} \frac{\cos \alpha}{S_{y^{\prime}}}\right)+X \frac{\sin \alpha}{S_{x}}-Y \frac{\cos \alpha}{S_{y}}
\end{aligned}
$$

Again these constants can easily be computed by the computer. Here the difference is that coordinates in the input image are calculated instead of in the output image. The way to create a complete output image is to calculate a corresponding coordinate pair in the input image for each coordinate pair in the output image. A raster-scan sequence is used, which gives some advantages for the design of the transformer. Let's start with $(x, y)=(0,0)$. The following points are $(0,1),(0,2)$, and so on, which results in the following scheme for the $i$ coordinate:

$$
\begin{aligned}
i_{0,0}= & E^{\prime}+0 \times B^{\prime}+0 \times A^{\prime} \\
i_{0,1}= & E^{\prime}+0 \times B^{\prime}+1 \times A^{\prime}=i_{0,0}+A^{\prime} \\
i_{0,2}= & E^{\prime}+0 \times B^{\prime}+2 \times A^{\prime}=i_{0,1}+A^{\prime} \\
& \cdots \\
i_{1,0}= & E^{\prime}+1 \times B^{\prime}+0 \times A^{\prime}=i_{0,0}+B^{\prime} \\
i_{1,1}= & E^{\prime}+1 \times B^{\prime}+1 \times A^{\prime}=i_{1,0}+A^{\prime} \\
& \cdots \\
i_{2,0}= & E^{\prime}+2 \times B^{\prime}+0 \times A^{\prime}=i_{1,0}+B^{\prime} \\
& \cdots
\end{aligned}
$$

As can be seen in the scheme above, the coordinates $(i, j)$ can be calculated incrementally.

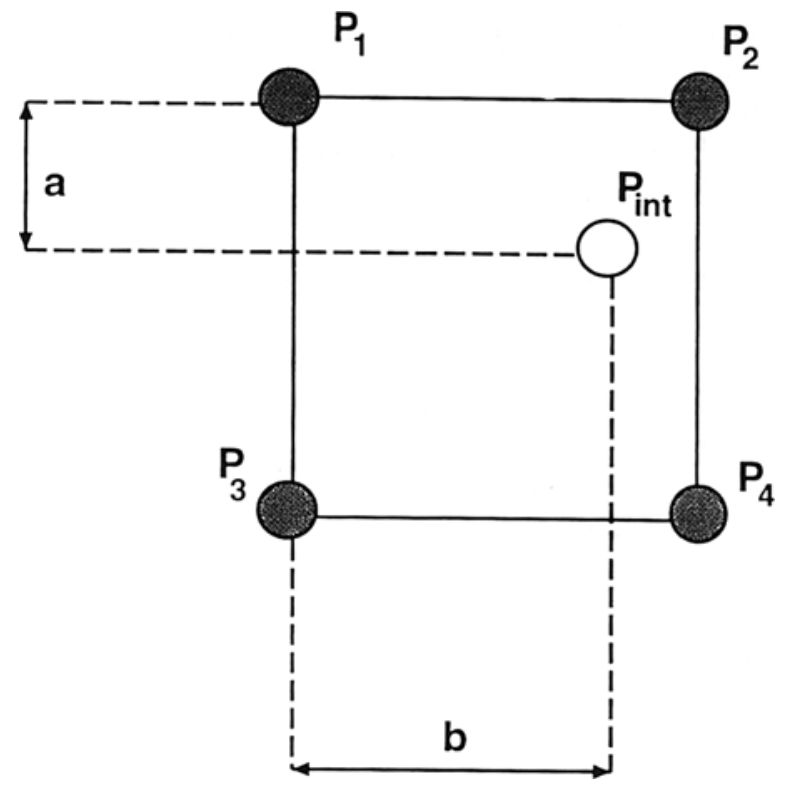

Fig. 3. Situation in output image where a pixel value has to be found in $P_{\text {int }}$.

Unfortunately the calculated coordinates in the input image will not likely be positioned on gridpoints, since the matrix multiplication in Eq. 3 yields coordinates with a fraction. The final image is an image with pixel values on a regular image matrix and therefore we need some kind of interpolation to find the actual pixel values. Figure 3 illustrates this situation. The points $P_{1}$ through $P_{4}$ are known pixel values and point $P_{\text {int }}$ is the point where we want to know the pixel value. If a Cartesian coordinate system is used with equal sized directions, the two-dimensional interpolation can be accomplished by a one-dimensional interpolation with respect to each coordinate axis [3]. Therefore we will only discuss the one-dimensional interpolation functions.

The ideal interpolation in one dimension applies an ideal low pass filter, which is a rectangular function in the frequency domain. In the spatial domain this corresponds to

$$
f\left(x_{\mathrm{int}}\right)=\sum_{k=-\infty}^{\infty} f\left(k x_{s}\right) \frac{\sin \left(\frac{\pi}{T_{c}}\left[x_{\mathrm{int}}-k x_{s}\right]\right)}{\left(\frac{\pi}{T_{c}}\left[x_{\mathrm{int}}-k x_{s}\right]\right)}
$$

with $f_{c}=T_{c}^{-1}$ being the cutoff frequency and $x_{s}$ being the sampling distance.

Resampling results in a repeated frequency spectrum. If the interpolation function is not an ideal filter, two effects might appear which can cause artifacts 
in the final image. First an attenuation of the higher frequencies in the original spectrum will occur. Secondly the repeated spectra will alias back into the original spectrum. Aliasing will only occur if the spectrum of the interpolation filter is not zero at frequencies above the cutoff frequency.

From Eq. 6 it follows that an infinite number of pixels should be used to calculate the new pixel value, which is of course impossible to realize. Therefore other interpolation techniques will have to be considered.

From a computational point of view, the easiest interpolation algorithm is the nearest neighbor interpolation, where each new pixel is given the value of the original pixel which is nearest to it. In the spatial domain this is equivalent to a convolution with a rectangle function. The frequency response is similar to a sincfunction. So higher frequencies are blurred and aliasing also occurs. In certain situations, such as diagonal straight lines, this causes some serious artifacts, like intensity steps instead of a continuous line. In medical applications this technique is not accurate enough.

Because of the disadvantages of nearest neighbor interpolation, a bilinear interpolation technique is often used. This technique calculates a new pixel value by a linear interpolation of the four surrounding pixel values. In the case shown in Fig. $3, P_{\text {int }}$ will be equal to:

$$
\begin{aligned}
P_{\mathrm{int}}= & (1-a)(1-b) P_{1}+(1-a) b P_{2} \\
& +a(1-b) P_{3}+a b P_{4} \quad(0 \leq a, b<1)
\end{aligned}
$$

Linear interpolation amounts to convolution of the sampled data with a triangle function. This triangle function corresponds to a modest low-pass filter in the frequency domain. Therefore it attenuates frequencies near the cut-off frequency resulting in smoothing of the image and it passes a significant amount of energy above the cut-off frequency.

In principle it is possible to use a higher order interpolation technique, where new points are calculated using at least four points in each direction. More information can be found in [3], [4] and [5]. Although higher interpolation techniques yields a higher quality of the images, the computational effort is significantly more complex. For this reason we have chosen to implement the bilinear interpolation technique.

\section{Technical Approach}

The problem of translating, rotating and scaling an image in real-time can be solved by dividing the system in two parts, a transformer and an interpolator. The image has a size of 1024 by 1024 pixels and the frame rate is at maximum 30 images per second (resulting in about $26 \times 10^{6}$ pixel operations per second). Furthermore, a total of 19 multiplications and additions (4 multiplications and 4 additions for the transformer and 8 multiplications and 3 additions for the bilinear interpolations) per pixel are required, which results in a computing requirement of 494 MOPS (Mega Operations Per Second). The use of lookup tables could possibly substantially lower this number, but it is still clear that computers do not have enough computing power to perform this task. Developing one or more ASICs is one solution. The idea is to make a printed circuit board (PCB) containing the ASICs and to put this board into a computer. A user interface is developed so the user can select the six constants $A^{\prime}$ through $F^{\prime}$ using mouse and keyboard. These constants are fed to the board every $25 \mathrm{~ms}$ (for each new frame). This task can easily be performed by a general purpose computer, not requiring the development of dedicated hardware.

Because the development of the transformer and interpolator are two different designs we will discuss them separately in the next two subsections. The third subsection will address the total design.

\subsection{Design of the Transformer}

If we look at Eq. 3 we see that there are two similar equations for $i$ and $j$. Therefore only one of the equations has to be implemented:

$$
i=A^{\prime} x+B^{\prime} y+E^{\prime}
$$

The screen scanning method, discussed in section 2 , will be implemented on a VLSI. In Fig. 4 a block diagram of this idea is given. The necessary multiplications are realized using one adder, saving silicon area. The operation is as follows (see Fig. 4): the $i$-reg register stores the computed address and the $Y \times B^{\prime}$-reg register saves the start value for each line. First the $E^{\prime}$ value is stored in $i$-reg and $Y \times B^{\prime}$-reg, which is the start value for the first line. Now the addresses of the original image for the first line are computed by the repetitive addition of $A^{\prime}$. After one line has been computed, $B^{\prime}$ is added to the previous start value for the computation of a line $\left(Y \times B^{\prime}\right)$ and saved in the $Y \times B^{\prime}$-reg register. Then the addresses in the original image for the next line will be computed by the incremental addition of $A^{\prime}$. At 


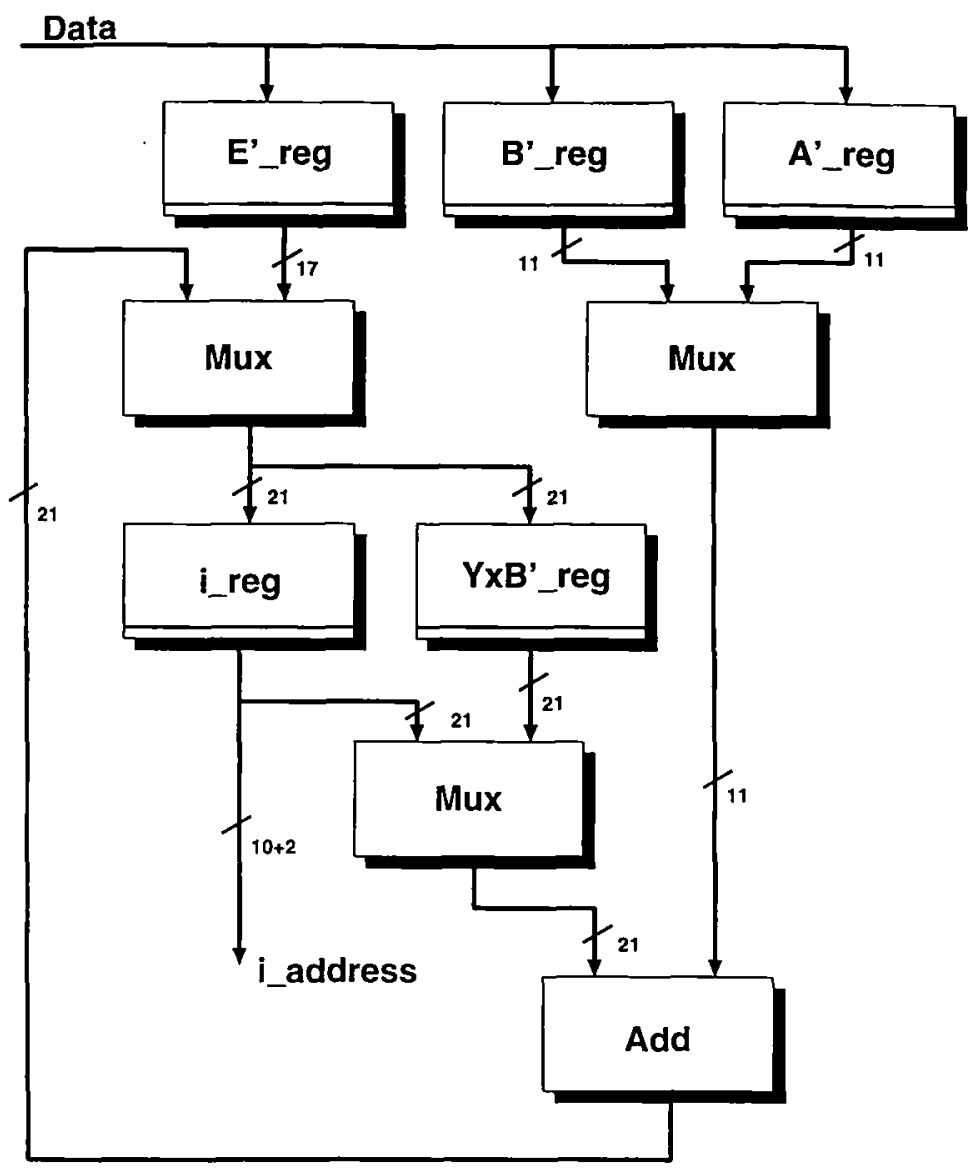

Fig. 4. Implementation of the transformer using an incremental computing scheme.

the end of the complete image $E^{\prime}$ is stored in the $Y \times B^{\prime}$-reg and $i$-reg register completing the rasterscan sequence.

This addressing scheme is implemented on a VLSI (actually two identical chips, one for the $i$ and one for the $j$ coordinate, resulting in a smaller chip with therefore a higher yield and lower cost). The $A^{\prime}$ and $B^{\prime}$ constants are products of zoomfactors and the cosine or sine of the angle (see Eq. 5). Therefore the zoomfactor determines the maximum value of these constants. Defining a minimum zoomfactor $S_{x}=S_{y}=\frac{1}{4}$, yields a maximum value of 4 for the two constants $A^{\prime}$ and $B^{\prime}$, which can be represented by a 3 bit integer part (with MSB being the sign bit) and an 8 bit fractional part (11 bits in total). The constant $E^{\prime}$ represents the translation of the output image with respect to the input image. Defining a maximal translation of a quarter of an image yields a 9 bit integer part (with one of them a sign bit) and a 8 bit fractional part; for a total of 17 bits for the $E^{\prime}$ constant. Intermediate calculations are done with 21 bits. The output address contains 10 bits for the integer part (to address between 0 and 1023) and 2 fractional bits.

\subsection{Design of the Interpolator}

As discussed before, it is very unlikely that the calculated coordinates in the input image fall onto gridpoints. However, the pixel values are only known on gridpoints. Therefore an interpolation technique is needed to obtain the corresponding pixel value. As discussed in section 2 we use the bilinear interpolation technique.

A direct implementation of the bilinear interpolation technique (Eq. 7) requires 8 multipliers and 3 adders (if we do not count the $(1-a)$ and $(1-b)$ calculations). Directly implementing these calculations in hardware will be difficult because of the speed constraint, similar to the problems with the transformer. Therefore other solutions were considered. 


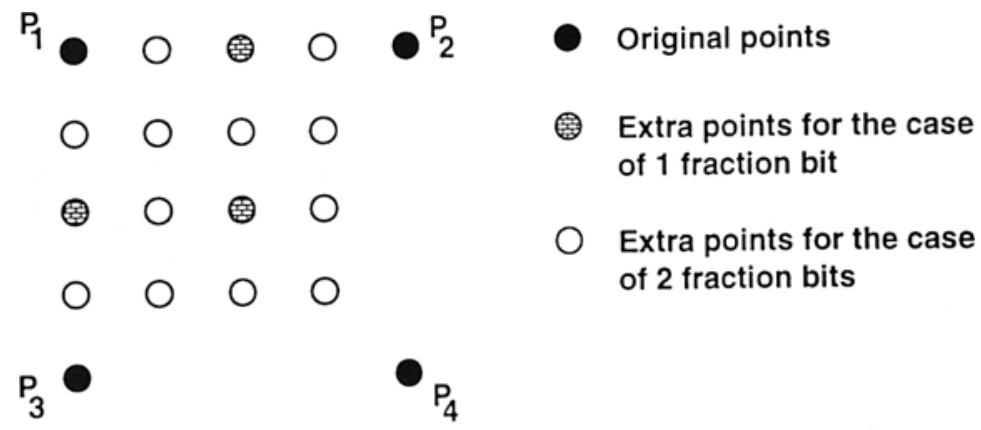

Fig. 5. Possible coordinates in case of two fraction bits.
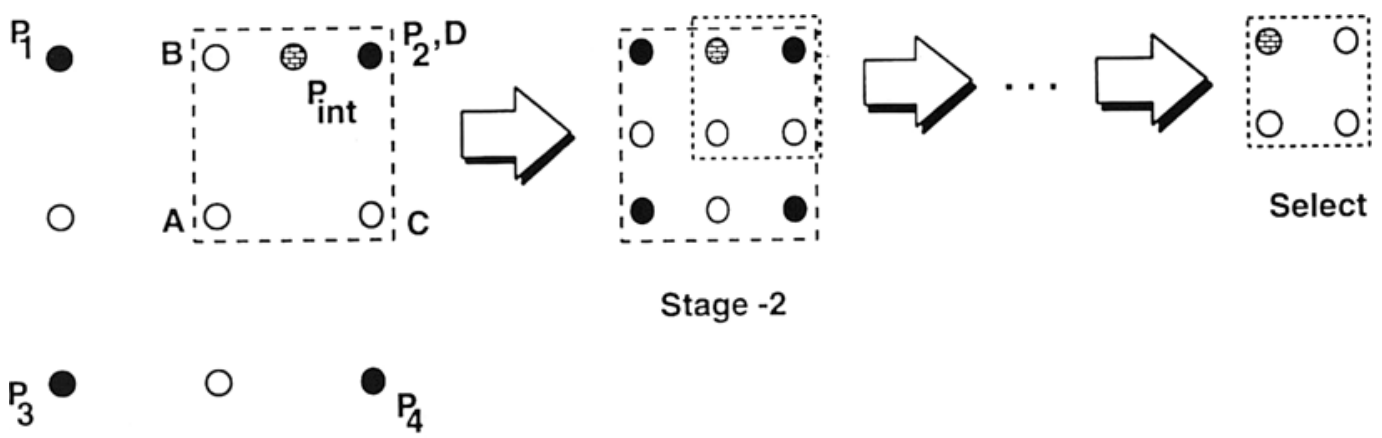

Stage -1

Fig. 6. Bilinear interpolation in stages.

The accuracy of the transformer determines the length of the fractional part of the coordinates (fixed point numbers are used for the coordinates instead of floating point numbers). Therefore only a limited number of coordinate values are possible. When two fraction bits are used, only the points shown in Fig. 5 are addressed, leading to a solution where multipliers can be avoided.

As stated in the introduction, the system described in this paper can be used in various applications and the accuracy demands of these applications differ. A medical application for instance requires much more accuracy than a consumer application like the digital video camera. A typical address accuracy of two bits is enough for such consumer applications, however, for medical application (e.g. dual energy image processing [4]) more than 5 fraction bits are sometimes required. Therefore flexibility in accuracy is required. We have designed a modular set up, so a flexible accuracy is easily achieved. A simple block for every fraction bit is designed. So, if four fraction bits are used, four building blocks will do the task. In Fig. 6 this idea is shown. In Fig. 7 a block diagram of a one-fraction-bit interpolation is given.

The bilinear interpolation is built up from different stages of these one-fraction-bit interpolations. In the first stage the intermediate points of the four known points are calculated, which is done by adding the values and doing one right shift operation (see block diagram in Fig. 7). Now the possible coordinates in the case of 1 fraction bit are calculated. The next thing to do is to select a quadrant where the requested pixel value is situated. This selection is accomplished by the values of the fraction bit in both directions. The quadrant is bounded by four new points which are the output values of this stage. Note that one of the four points is an original pixel value. If another fraction bit is available, another stage is added, using the output values of the previous stage as input values. The four output values of the last stage will be the input values of the 

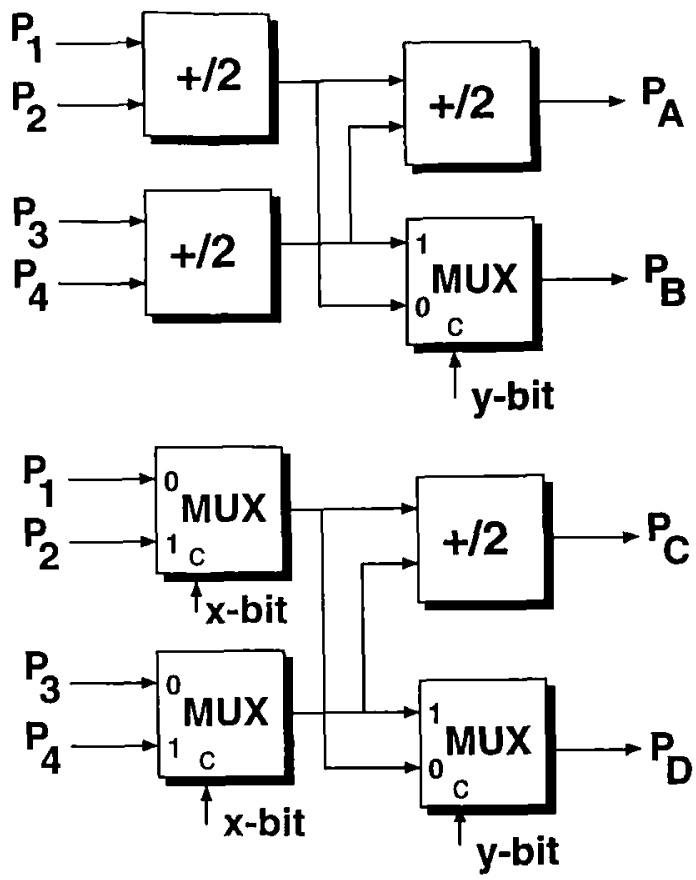

Fig. 7. Block diagram of an one-fraction-bit interpolation.

selection unit, which will select the final value. By implementing this modular setup which uses computed intermediate values, only adders and no multipliers are needed, saving silicon area.

In Fig. 8 a block diagram of the bilinear interpolation is given. If more accurate interpolation is required, another ASIC (containing an interpolation stage) is used until the required accuracy is achieved.

\subsection{Design of the Total System}

Now that the transformer and the interpolator have been discussed, the total system will be addressed. Two transformers are used, which calculate coordinates in the input image. If the calculated coordinates are not in the address range of the input image, the interpolation is skipped and the output pixel becomes zero (black when using a standard colormap). Using the four surrounding pixel values, the final value in the output image is calculated using the modular bilinear interpolator. Every $25 \mathrm{~ns}$ a pixel value is calculated in the output image. Using conventional memory architecture and one access per pixel requires a memory pixel rate of $25 / 4=6 \mathrm{~ns}$. Because memory with a pixel rate of $6 \mathrm{~ns}$ is extremely expensive (if it is even available), therefore four mernory banks are used instead of one. The pixels are stored in such a fashion that only one value from each RAM is needed for every pixel in the output image. This can be achieved by making the following division:

RAM1: Pixels from even lines and even columns

RAM2: Pixels from even lines and odd columns

RAM3: Pixels from odd lines and even columns

RAM4: Pixels from odd lines and odd columns

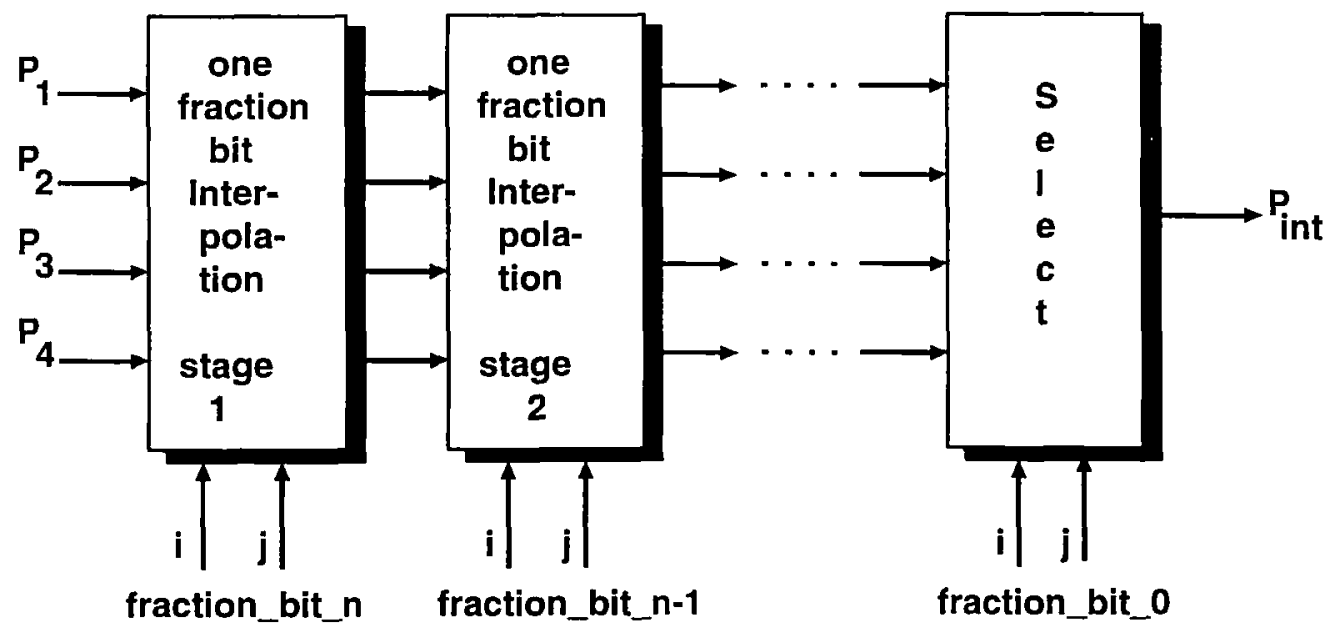

Fig. 8. Block diagram bilinear interpolation. 


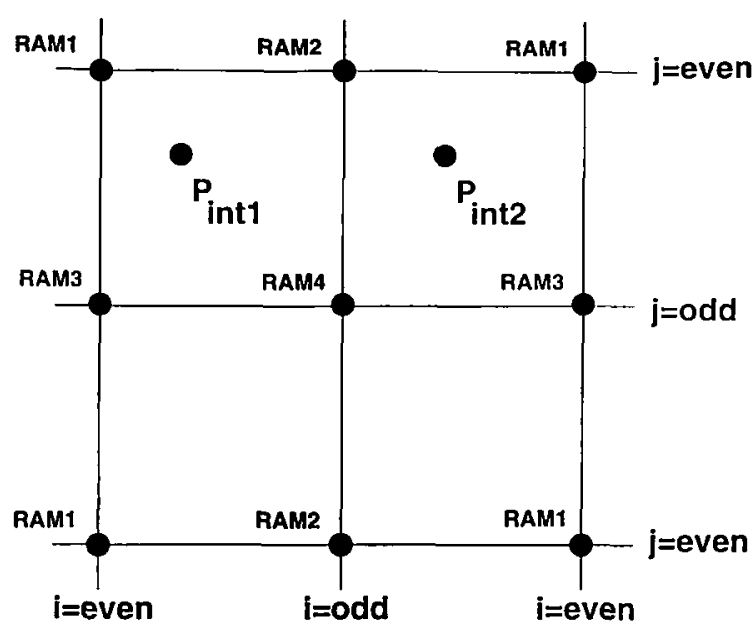

Fig. 9. Illustration of the need for extra selection logic.

Note that ihe total amount of memory is the same as in the case where one single memory is used, but that the memory bandwidth is four times higher. The total amount of RAM is $1024 \times 1024$ pixels $\times 8$ bits $\times 2$ (We use a so-called ping-pong memory: One memory is used to write new data in, while another memory is used for display) $=2$ MByte.

The disadvantage of this memory distribution scheme is that some RAM selection logic must be added. Figure 9 illustrates the need for the extra RAM selection logic. In this figure a part of the source image can be seen. The distribution of the pixel values along the RAMs is also depicted. For $P_{\mathrm{int} 1}$ the upper left corner is a value out of RAM1. The values out of the other RAMs can be selected by the same coordinate pair. For $P_{\text {int } 2 \text {, however, this situation is }}$ different. Rounding the coordinate of $P_{\text {int 2, yields the }}$ correct address for RAM2 and RAM4. For the correct address of RAM1 and RAM3, a coordinate pair which is one position higher than the coordinate pair for RAM2 and RAM4 is needed. The same discussion holds for situations in the other direction. Therefore some logic is needed to control the correct addresses for the RAMs. Since the situation depends on the value of the computed address (odd or even) the control logic is very easy to design.

In Fig. 10 the setup of the total system is shown. A Personal Computer with a user interface generates a few control signals for the system as well as the 6 constants $A^{\prime}$ through $F^{\prime}$. Two transformers (ASIC number 1) calculate the coordinates in the source image. If the calculated address is out of the address range of the input image, a white pixel is sent to the output image. With some selection logic the four surrounding known pixel values from the source image are fetched and used in the second ASIC, the interpolator.

\section{Design Methodology}

When developing the algorithm and the organization of the system all possible configurations should be taken into account in order to take full advantage of the use of ASICs. A high-level description of the ASICs and the total system clarifies the interfaces and the functionality of the different parts and has often proved to be necessary to guarantee a first-time-correct design. In our case, the high-level description was made using MoDL [6] which offers similar (and more) facilities to VHDL. With this language the total image processing system was described: an image was taken as input, the memory organization was tested, the developed algorithm (divided over several ASICs) was executed and a bitmap as output was obtained. Using this bitmap output, errors could be visualized much easier compared to general test vectors.

After the high-level description was finished and proven to be correct (with the help of the algebraic simulation capability of MoDL) the design team split in different groups [7]. One group designed the interpolator ASIC while another group developed the transformer ASIC. A third group designed the PCB. Using the pertinent high-level description as a reference, each group could develop their design towards gate level. A major aspect during this detailing/refinement process was the efficient mapping of the algorithm onto the hardware resources. If an alteration of the algorithm would give a better use of the hardware resources a modification and test of the system was performed. The high-level description served as a reference to which the detailed designs could be compared. See also Fig. 11.

The designs were realized in a $1.5 \mu \mathrm{m}$ double metal single poly CMOS standard cell technology. Schematic entry of the designs was done with Mentor Graphics' IDEA Station [8]. The designs were simulated with Quicksim [9] and results were compared with the high-level description to ensure a functionally correct design.

In the design process, the algorithms were mapped on the available standard cells. Here the designer tried to make this mapping as efficient as possible taking into account the delay of the different standard cell 


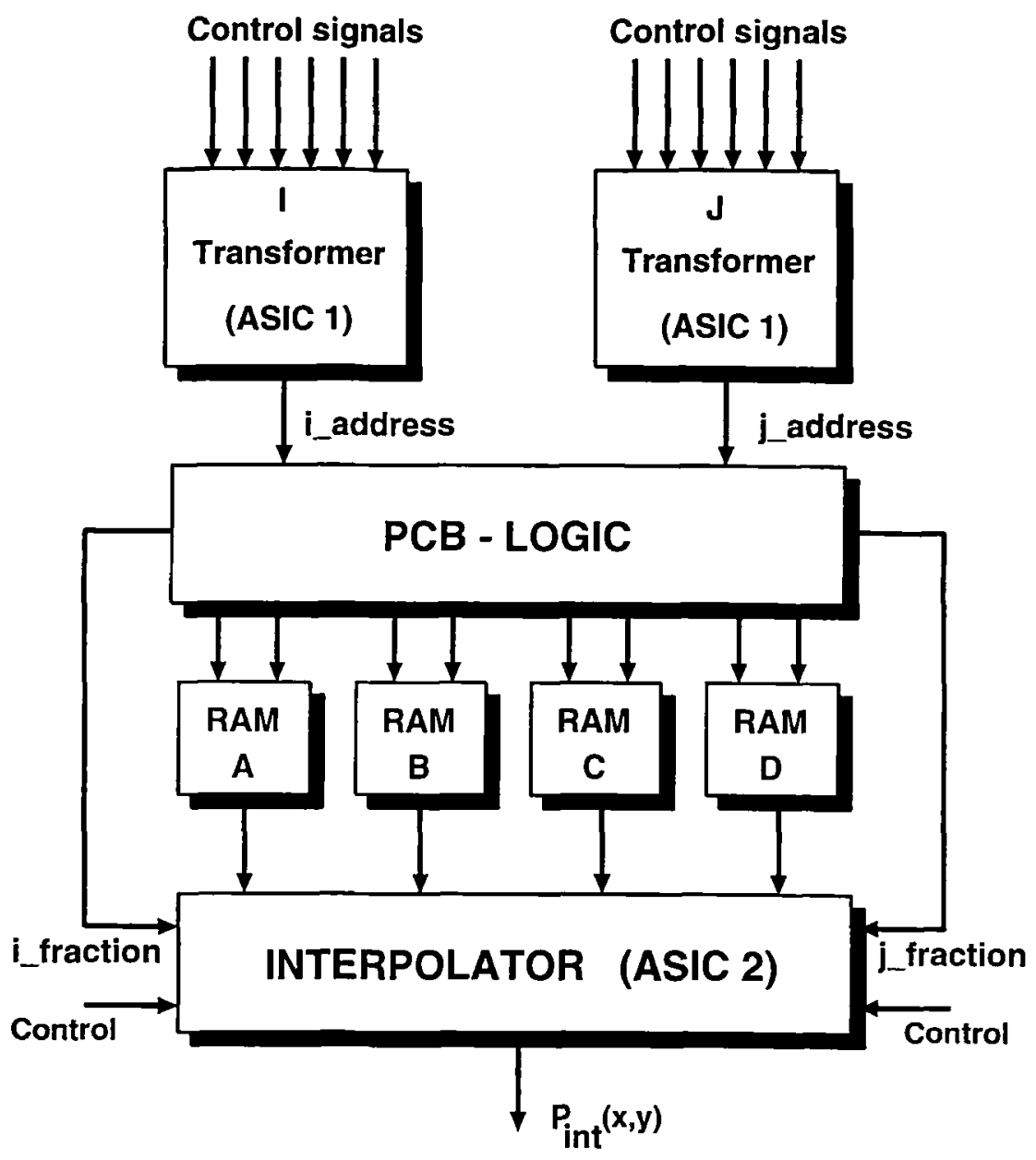

Fig. 10. Block diagram of the total system.

components, which led to a recursive process of design changes until the timing requirements were met. When the schematic design was correct the layout generation was done using Mentor Graphics' CELL Station [10]. After the generation of the layout the two ASICs were processed at the foundry. The last step was the design of the printed circuit board for the total system. This PCB contains three of the designed ASICs, a transformer ASIC for both the $i$ - and $j$-address and an interpolator ASIC performing a two step interpolation. Additionally some control logic with the frame and line synchronization data and the system clock were required.

\section{Results}

The total design resulted in a system which performs the translation, rotation and scaling operation at a speed of 25-30 frames per second. Each computed image is interpolated in two steps using a bilinear interpolation technique. By dividing the original image over four memories, the memory bandwidth is limited so expensive high speed memory is not needed. With the computed address four surrounding pixel values are selected from each of the four RAMs. The resulting pixel value after interpolation is stored in a (video) memory. We specified and simulated the total system using a high-level design language (in our case MoDL [6]). From this description we derived a hardware description of the separate ASICs from which the ASICs could be realized. At this moment the ASICs have been processed and the PCB design is ready. The ASICs as well as the memory and the controller (implemented in FPGA) fit on a PC-AT board. In Table 1 the most important parameters of the ASICs are presented. The 


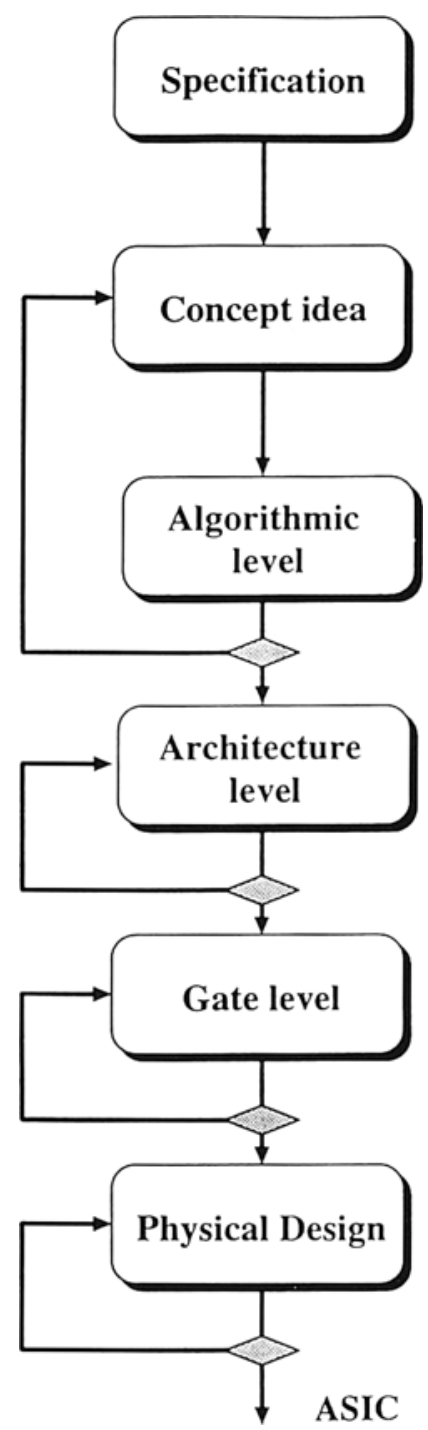

Start with the specification

of the system.

After several ideas a concept

idea is born.

Description of the complete system

and simulation with a test image.

In this level also the dividing in subtasks is done.

Sequential description of the ASIC (and later concurrent) and simulation with a test image.

Realization of the ASIC with a design tool (i.e. Mentor Graphics Cell Station).

Submission of the tape of the layout to the foundry.

Fig. 11. Design flow.

clock speed in this table is the clock speed needed for manipulating a $1024 \times 1024$ images with a $40 \mathrm{~ms}$ latency ( 25 images per second). In Fig. 12 the floorplans of the two ASICs is shown and in Fig. 13 a photograph of the PCB is shown. The socket of the interpolation chip did not fit on the PCB, so an alternative

Table 1. Parameters of the designed ASICs.

\begin{tabular}{lcc}
\hline & Transformer & Interpolator \\
\hline Area & $2.93 \times 3.15 \mathrm{~mm}^{2}$ & $3.52 \times 3.55 \mathrm{~mm}^{2}$ \\
Clock speed & $40 \mathrm{MHz}$ & $40 \mathrm{MHz}$ \\
Pin count & 34 pins & 51 pins \\
\hline
\end{tabular}

home-made socket has been used, as can be seen on the photograph. The system is completely functional and meets the specifications.

\section{Discussion}

Starting the design process from a system point of view, we were able to organize the system in such a way that expensive hardware was avoided. The use of adders instead of multipliers proved to be sufficient here. A thorough survey of the system partitioning was made beforehand. 


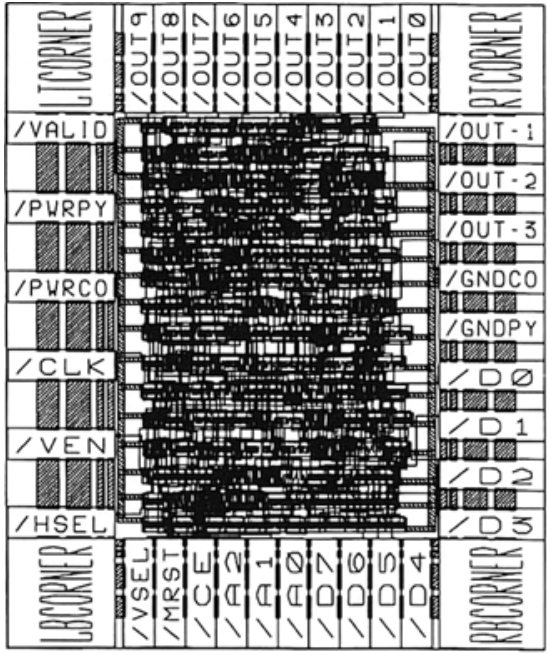

Transformer

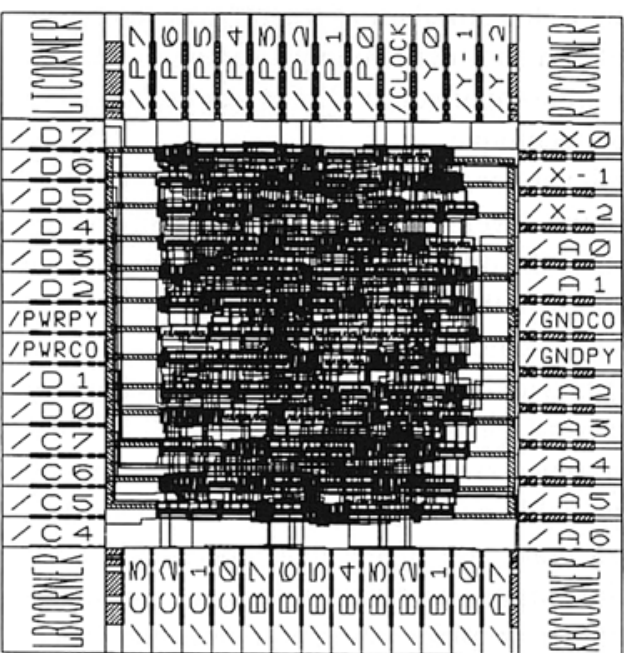

Interpolator

Fig. 12. Floorplans of the ASICs.

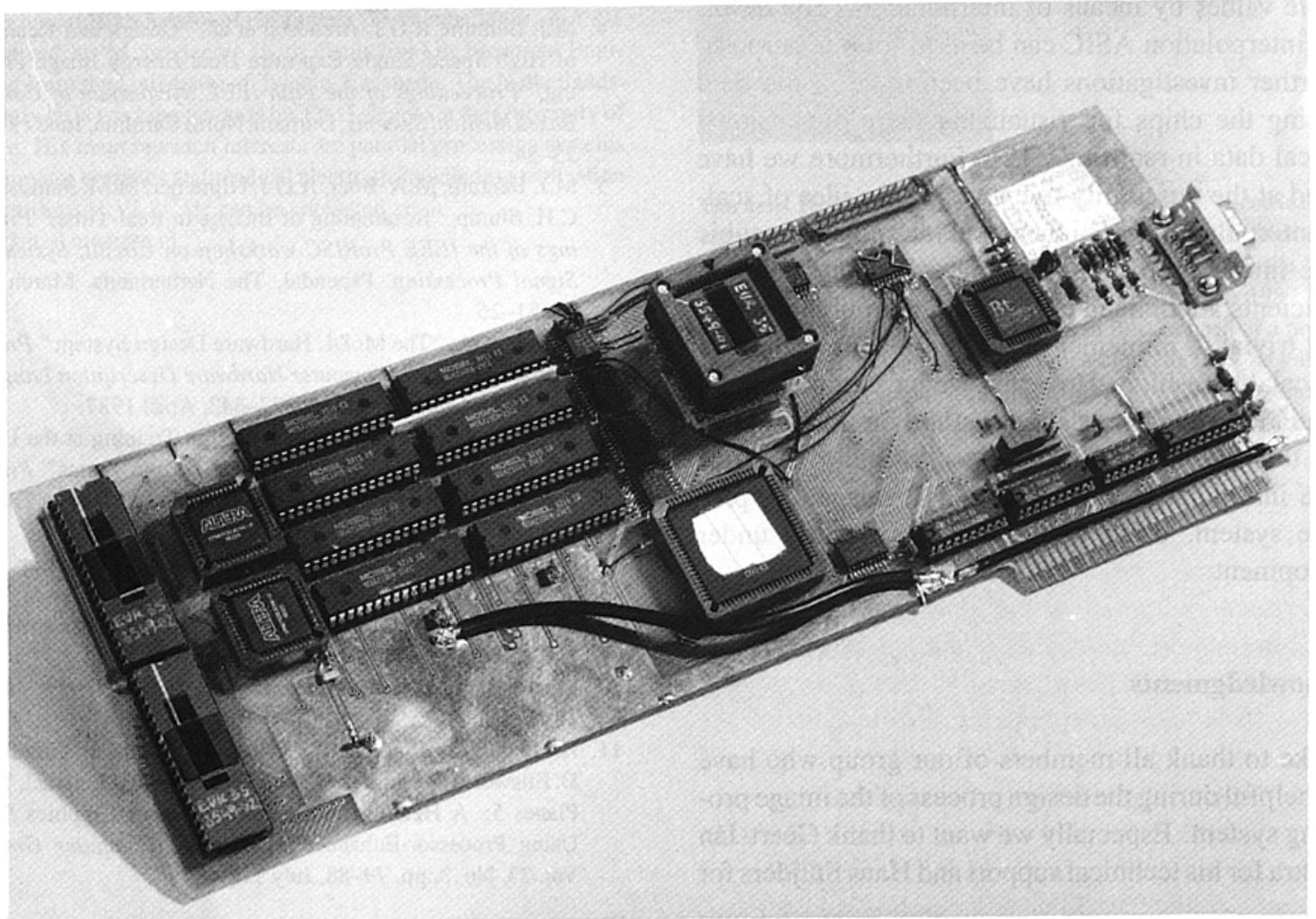

Fig. 13. The printed circuit board. 
According to the simulations the bilinear interpolation resulted in a considerable improvement in the image quality, in comparison with the nearest neighbor interpolation technique.

The high-level description used was found to be of great help in the development of the algorithm and the specification of the different interfaces. In this way an efficient solution to the problem and a first-time-correct design could be achieved.

The described architecture is not only interesting for image processing applications, but also for (real-time) computer graphics systems. Two algorithms, heavily used in this field, color interpolation and Gouraud Shading, can make use of the developed ASICs. Of course special purpose hardware is designed especially for the computer graphics systems, like in the PixelPlanes System [11]. The main difference between the interpolator we discussed in this paper and other implementations is that our solution does not make use of multipliers. This results in a small chip area. Another application field is the visualization of three dimensional data. In this visualization step we want to map a three dimensional scene onto a two dimensional screen [12]. One step in this algorithm is the calculation of sample values by means of interpolation. The developed interpolation ASIC can be used for this purpose.

Further investigations have been done in the field of using the chips for visualizing three dimensional medical data in real-time [12]. Furthermore we have looked at the possibility to extending the idea of scalable interpolation to a higher level interpolation: cubic spline interpolation. Since cubic spline interpolation coefficients are defined by a third order polynomial, it is not trivial to implement the interpolation technique in a scalable fashion. However, we have implemented the cubic spline interpolation method on a VLSI [13]. With this ASIC it is possible to do real-time cubic spline interpolation on a $512 \times 512$ image. A prototype system, using this ASIC, is currently under development.

\section{Acknowledgments}

We like to thank all members of our group who have been helpful during the design process of the image processing system. Especially we want to thank Geert-Jan Laanstra for his technical support and Hans Snijders for his software and design support. Furthermore we want to thank the students Mark Boerrigter, Marco Bosma, Durk van Veen, Marc Weusting and Nam Tran for making the layouts of the ASICs and the PCB.

We also like to thank the anonymous referees for their valuable comments which, we have convinced, have improved the clarity of the presentation and have pointed out interesting related work.

Finally we would like to thank the Eurochip VLSI Design Training Action for supplying CAD-software and processing facilities.

The investigations were partly supported by the foundation for Computer Science in the Netherlands (SION) with financial support from the Netherlands Organization for Scientific Research (NWO).

\section{References}

1. M.P. Ekstrom, Digital Image Processing Techniques, Orlando, FL: Academic Press, Chapter 8, p. 298, 1984.

2. Proceedings of the 11th International Conference on Pattern Recognition, The Hague, The Netherlands, September 1992, sessions A3, A9 and A14.

3. J.A. Parker, R.V. Kenyon, and D.E. Troxel, "Comparison of Interpolating Methods for Image Resampling," IEEE Transaction. on Medical Imaging, Vol. 1, pp. 31-39, 1983.

4. M.J. Bentum, R.G.J. Arendsen et al., "Design and Realization of High Speed Single Exposure Dual Energy Image Processing," Proceedings of the Fifth IEEE Symposium of Computer Based Medical Systems, Durham, North Carolina, June 1992, pp. 25-34.

5. M.J. Bentum, M.A. Boer, A.G.J. Nijmeijer, M.M. Samsom, and C.H. Slump, "Resampling of Images in Real-Time," Proceedings of the IEEE ProRISC workshop on Circuit, Systems and Signal Processing, Papendal, The Netherlands, March 1994, pp. 21-26.

6. J. Smit et al., "The MoDL Hardware Design System," Proceedings 8th Int. Conf. Computer Hardware Description Languages and Their Applications, pp. 327-342, April 1987.

7. M.M. Samsom, "VLSI System Design Training at the University of Twente by Means of Student Design Teams," Proceedings of the Third Eurochip Workshop on VLSI Design Training, Grenoble, France, October 1992, pp. 66-71.

8. Mentor Graphics, IDEA Series Schematic Capture User's Manual, 1989/1990.

9. Mentor Graphics, IDEA Series Quicksim User's Manual, $1989 / 1990$.

10. Mentor Graphics, IDEA Series Cell Station User's Manual, $1989 / 1990$.

11. H. Fuchs, J. Poulton, J. Eyles, T. Greer, J. Goldfeather, D. Ellsworth, S. Molnar, G. Turk, B. Tebbs, and L. Israel, "PixelPlanes 5: A Heterogeneous Multiprocessor Graphics System Using Processor-Enhanced Memories," Computer Graphics, Vol. 23, No. 3, pp. 79-88, July 1989. 
12. M.J. Bentum and J. Smit, "Design of a Parallel VLSI Engine for Real-Time Visualization of 3D Medical Images," Proceedings of SPIE Medical Imaging 1994, Newport Beach, California, February 1994, Vol. 2164, pp. 370-381.

13. A.G.J. Nijmeijer, M.A. Boer, C.H. Slump, M.M. Samsom, M.J. Bentum, G.J. Laanstra, J. Smit, and O.E. Herrmann, "Correction of Lens-Distortion for Real-Time Image Processing Systems," Proceedings of the 1993 IEEE Workshop on VLSI Signal Processing. Veldhoven, The Netherlands, October 1993, pp. 316-324.

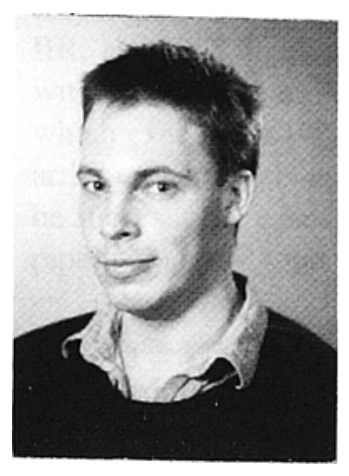

Mark J. Bentum was bom in Smilde, The Netherlands in 1967. In 1988 he received the B.S. degree in Electrical Engineering from the Polytechnical High School of Groningen, The Netherlands. In 1991 he received his M.Sc. degree (with distinction) in Electrical Engineering from the University of Twente, Enschede, The Netherlands. Currently he is working towards his $\mathrm{Ph}$.D. degree at the University of Twente. His main research interests are parallel processing systems for computer graphics and medical electronics, including realization of algorithms in VLSI. He can be contacted at:

mark@nt.el.utwente.nl

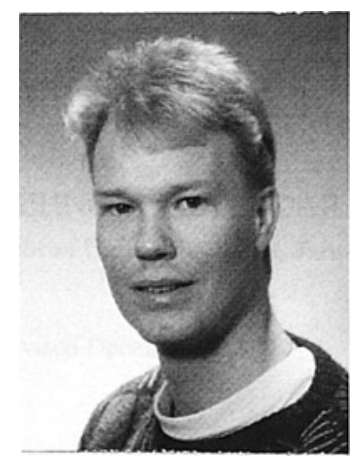

Martin M. Samsom was born in Amsterdam, The Netherlands in 1966. In 1989 he received his M.Sc. degree in Electrical Engineering from the University of Twente, Enschede, The Netherlands. Currently he is working as a lecturer in VLSI System Design. His main interests are VLSI System Design, High Level Description Methods and Digital Signal Processing.

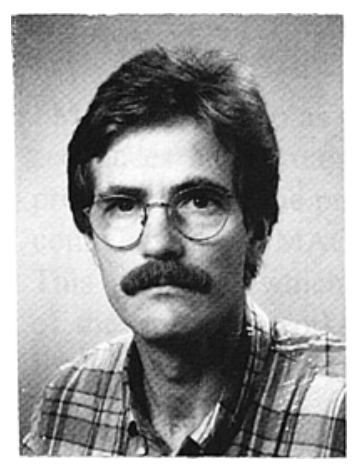

Cornelis H. Slump received the M.Sc. degree in Electrical Engineering from Delft University of Technology, Delft, The Netherlands in 1979. In 1984 he obtained his Ph.D. in physics from the University of Groningen, The Netherlands. From 1983 to 1989 he was employed at Philips Medical Systems in Best as head of a predevelopment group on medical image processing. In 1989 he joined the Network Theory group from the University of Twente, Enschede. The Netherlands. His main research interest is in digital signal processing, including realization of algorithms in VLSI. 